\title{
Anatomical factors influencing development of trichiasis and entropion in trachoma
}

\author{
Susan Lewallen, Paul Courtright
}

\begin{abstract}
There has been no published investigation into the reasons why some patients with severe trachomatous conjunctival scarring develop entropion and trichiasis while others do not. In a population of leprosy patients with severe trachomatous conjunctival scarring the authors found that lagophthalmos correlated with the absence of trichiasis and entropion. We suggest that orbicularis oculi muscle function, as well as other anatomical variations among individuals, may contribute to the development of trachomatous entropion and trichiasis in patients with conjunctival scarring.
\end{abstract}

Trachoma remains the leading cause of preventable blindness worldwide. ${ }^{1}$ Blindness is secondary to corneal scarring caused by chronic entropion and trichiasis, which are associated with scarring of the superior tarsal conjunctiva after chronic infection with Chlamydia trachomatis. However, not everyone who is infected with the causative agent becomes blind. Some investigators have suggested that trachoma may be an immunopathological disease, and that repeated reinfection with the causative organism, perhaps exacerbated by superinfection with other organisms, is necessary to cause severe conjunctival scarring. ${ }^{23}$

Another issue in the development of trachoma blindness is why only some of the patients who have severe scarring of the conjunctiva develop entropion and trichiasis. The magnitude and cause of this variability have not been established. As part of a separate study on cellular response and conjunctival scarring we examined leprosy patients in a trachoma endemic region of Ethiopia. We noticed that there was less trichiasis/entropion among leprosy patients with lagophthalmos than among those who had normal orbicularis function. Leprosy patients often have weakness of the orbicularis oculi muscle, and we considered whether this factor might influence the development of entropion. Thus we analysed relevant data collected to examine this hypothesis.

International Centre for Eye Health, 27-29 Cayton Street, London EC1V 9E P Courtright

Reprints from: Professor Gordon J Johnson, International Centre for Eye International Centre for Eye London ECIV $9 \mathrm{EJ}$.

Correspondence to: Dr Susan Lewallen, PO Box 2273, Blantyre Malawi.

Accepted for publication 4 April 1991

\section{Materials and methods}

Leprosy patients from the Sheshamane area of South Shoa province in Ethiopia, where trachoma is hyperendemic, were examined from February to May 1990. One examiner (SL) conducted all the examinations and recorded the following data for each patient: age, sex, conjunctival scarring, trichiasis/entropion, and presence of lagophthalmos. Conjunctival scarring and trichiasis/entropion were graded accord- ing to the WHO (non-simplified) grading scheme. ${ }^{1}$ Eyes with severe (grade 3 ) conjunctival scarring were used for analytic purposes.

Data were entered on a computer and analysed in a $2 \times 2$ table by Fisher's exact test and multiple logistic regression analysis. Since lagophthalmos may be unilateral the data were analysed by eye rather than by individual.

\section{Results}

Thirty seven patients (53 eyes) had severe conjunctival scarring. The ages of the patients ranged from 33 to 75 years, median 50 . None of these patients had leprosy related loss of eyelashes. One patient with trichiasis/entropion due to a leprosy nodule on the lid was excluded from the analysis. We chose the eye with severe scarring in cases of monocular scarring $(n=19)$ and the left eye was chosen in cases of binocular severe scarring $(n=18)$.

Of the 37 patients with severe conjunctival scarring 16 had lagophthalmos. Only one of these patients had coexisting grade 3 trichiasis/ entropion. In contrast, 21 patients with severe scarring had no lagophthalmos, and nine of these had coexisting grade 3 trichiasis/entropion (Table 1). Logistic regression analysis revealed that neither age nor sex confounded the association between lagophthalmos and grade 3 trichiasis/entropion. The average age of patients with trichiasis/entropion was the same ( 51 years) as that of patients without trichiasis/entropion.

\section{Discussion}

Since entropion and trichiasis are frequently the cause of the blindness that results from trachoma it is relevant to ask why some patients with conjunctival scarring develop this while others do not. Lagophthalmos appears to be a factor protecting some leprosy patients with trachomatous conjunctival scarring from developing entropion. Obviously in the non-leprosy population lagophthalmos itself is not common enough to be proposed as a significant protective factor.
Table 1 Presence of trichiasis/entropion among patients with severe conjunctival scarring

\begin{tabular}{llll}
\hline & \multicolumn{3}{c}{ Number of patients with trichiasis/entropion } \\
\cline { 2 - 4 } & Grade 0-1 & Grade 2 & Grade 3 \\
\hline No lagophthalmos & 9 & 3 & 9 \\
Lagophthalmos & 13 & 2 & 1 \\
\hline
\end{tabular}

Correlation: Grade 3 (severe) trichiasis/entropion: odds ratio= 0.09 ( $95 \%$ confidence interval: $0.01,0.89)$; Fisher's exact onetailed $\mathrm{p}$ value $=0.01$. Grades $2+3$ (moderate + severe) trichiasis/ entropion: odds ratio $=0 \cdot 17$ ( $95 \%$ confidence interval: $0.03,0.96)$; Fisher's exact one-tailed $p$ value $=0 \cdot 02$ 
However our findings suggest that anatomical variations among individuals, specifically orbicularis oculi strength, may influence the development of entropion and trichiasis in the face of severe conjunctival cicatrisation. Deep insertions of the pretarsal orbicularis within the orbital rim allow a posterior pull on the eyelids to maintain apposition to the globe. The relative strength of marginal fibres of the orbicularis in comparison with the rest of the pretarsal or preseptal orbicularis may influence the likelihood of the tarsus rotating inward. The development of involutional entropion of the lower lid is thought to be related to such anatomical factors. ${ }^{4}$ Some of these same factors may play a role in development of trichiasis/entropion once cicatrisation of the conjunctiva has occurred.

These data were not collected for the purpose of assessing the correlation between lagophthalmos and trichiasis/entropion and there are some limitations to our using them thus. Our findings are limited by the use of the WHO grading system for trichiasis/entropion, which is a better measure of trichiasis than of the entropion. To examine the role of an individual's lid anatomy in the development of entropion it would be preferable to grade lids according to a system which separates the degree of entropion and lid deformity from the number or position of inturn- ing lashes. Such a system was suggested by Sarkies in $1965 .^{5}$ The presence of lagophthalmos is a gross measure of orbicularis strength, and obviously lagophthalmos is not the factor protecting patients with severe scarring in thë onleprosy population from developing entropion. However, orbicularis strength may be an important risk factor, as may other variations in individual lid anatomy. Further investigations would help clarify the pathogenesis of blinding trachoma and prove useful in enhancing the identification of patients at highest risk of blindness from this disease.

This investigation received financial support from the UNDP/ World Bank NWHO Special Programme for Research and Training in Tropical Diseases (TDR). Dr Lewallen was supported by Christoffel Blindenmission. The authors acknowledge the support of the Ministry of Health, Addis Ababa, Ethiopia.

1 Dawson CR, Jones BR, Tarizzo ML. Guide to trachoma control. Geneva: World Health Organisation, 1981: 16

2 Grayston JT, Wang SP, Yeh LJ, Kuo CC. Importance of reinfection in the pathogenesis of trachoma. Rev Infect Dis $1985 ; 7: 717-25$.

3 Williams DM, Schachter J. Role of cell-mediated immunity in chlamydial infection: implications for ocular immunity. Rev Infect Dis 1985; 7: 754-9.

4 Jones JT. The anatomy of the lower eyelid, and its relations to the cause and cure of entropion. Am $\mathcal{F}$ Ophthalmol 1960; 49: the cause

5 Sarkies JWR. Early changes in margin of upper eyelid in entropion complicating trachoma. Brf Ophthalmol 1965; 49: 538-41. 